\title{
Remisión de la psoriasis luego de bypass gástrico*
}

\author{
Drs. MARÍA MAGDALENA FARÍAS N. ${ }^{1}$, MARCELA CONCHA R. ${ }^{2}$, CLAUDIA DE LA CRUZ F. ${ }^{2}$ \\ 1 Departamento de Nutrición. \\ 2 Departamento de Dermatología. \\ Pontificia Universidad Católica de Chile, Santiago, Chile.
}

\begin{abstract}
Psoriasis remission after gastric bypass

Psoriasis is a frequent skin disease affecting 1-3\% of the population. Recent studies have found that psoriasis is linked to higher rate of obesity, and that obesity itself is a risk factor for the development and severity of psoriasis. The common link between psoriasis and obesity may be a state of chronic inflammation, including elevated serum levels of cytokines such as tumor necrosis factor. Consequently, weight loss has been proposed as a useful adjunctive therapy for obese patients with psoriasis. We present a 43 -year old man with body mass index greater than $40 \mathrm{~kg} / \mathrm{m}^{2}$ who had marked improvement in his psoriasis after Roux-en-Y gastric bypass surgery. Weight loss following bariatric surgery could play a role on the treatment for psoriasis on morbid obese patients.
\end{abstract}

Key words: Psoriasis, bariatric surgery, gastric bypass, weight loss.

\section{Resumen}

La psoriasis es una patología dermatológica frecuente que afecta entre 1 al 3\% de la población. Estudios recientes han identificado mayor índice de obesidad en los pacientes con psoriasis. También se ha reportado que la obesidad, por si sola, constituye un factor de riesgo, tanto del desarrollo como de la severidad, de la psoriasis. Ambas enfermedades estarían ligadas por un estado inflamatorio crónico caracterizado por aumento de los niveles plasmáticos de citoquinas, tal como el factor de necrosis tumoral. Consecuentemente, la baja de peso se ha propuesto como una herramienta complementaria al tratamiento de los pacientes obesos con psoriasis. Se presenta un paciente de sexo masculino, de 43 años de edad, con índice de masa corporal mayor a $40 \mathrm{~kg} / \mathrm{m}^{2}$, quien presentó una marcada mejoría de su psoriasis luego de la realización de un bypass gástrico. El tratamiento de la obesidad por medio de la cirugía bariátrica podría tener un rol en el manejo de la psoriasis en pacientes con obesidad mórbida.

Palabras clave: Psoriasis, cirugía bariátrica, bypass gástrico, baja de peso.

*Recibido el 3 de mayo de 2011 y aceptado para publicación el 12 de septiembre de 2011.

Trabajo sin financiamiento ni conflicto de interés.

Correspondencia: Dra. M. Magdalena Farías N.

Albacete 4420, Dep. 56, Santiago, Chile.

mmfarias@gmail.com 


\section{Introducción}

La psoriasis es una dermatosis inflamatoria caracterizada por lesiones eritematoescamosas que afecta al 1-3\% de la población, dependiendo de la región geográfica y la raza. Su etiopatogenia no está del todo elucidada, sin embargo, se identifica un sustrato genético, así como factores ambientales e inmunológicos. La enfermedad afecta por igual a ambos sexos y tiene una marcada predisposición familiar ${ }^{1}$.

Sus manifestaciones clínicas son heterogéneas, desde lesiones leves hasta formas muy extensas. En cualquiera de sus formas, tiene un gran impacto negativo en la calidad de vida. Dado su carácter crónico y recurrente, constituye un gran desafío terapéutico en Dermatología ${ }^{2}$.

Recientemente, a través de estudios epidemiológicos, se ha identificado un mayor riesgo de desarrollar algunas alteraciones metabólicas en los pacientes con psoriasis, dentro de las cuales destaca la obesidad ${ }^{3}$. Ambas patologías estarían vinculadas mediante un mecanismo fisiopatológico común que se explica por una inflamación crónica de bajo grado ${ }^{4}$. No sólo la obesidad se asocia a una mayor incidencia y severidad de la psoriasis, sino que también afecta de manera negativa su respuesta al tratamiento 5 .

Se ha observado que los síntomas de la psoriasis pueden mejorar considerablemente en pacientes que pierden peso $^{6}$, por lo que el manejo de la obesidad se plantea como una posible herramienta terapéutica para la psoriasis, o al menos, que permita una mayor respuesta a la terapia convencional ${ }^{7}$.

\section{Caso clínico}

Se presenta un paciente de sexo masculino de 43 años de edad, obeso, hipertenso, diabético tipo 2, el cual consultó en nuestro Servicio de Dermatología en junio de 2007 por presentar psoriasis mal controlada de 10 años de evolución. Al examen físico presentaba obesidad de distribución abdominal. En el examen dermatológico destacaban placas eritematosas intensamente infiltradas y descamativas, en codos, rodillas, piernas, cuero cabelludo, zona lumbar y manos, además de una queratodermia palmoplantar. Inicialmente se trató con medicamentos tópicos (corticoides y queratolíticos), con mejoría intermitente de sus lesiones, pero sin lograr una respuesta satisfactoria ni mantenida en el tiempo. Tres años más tarde se decidió tomar una conducta más agresiva e indicar un tratamiento sistémico con un retinoide oral (Neotigasón ${ }^{\circledR} 25 \mathrm{mg}$ al día), sin respuesta inicial por lo que se aumentó la dosis hasta $75 \mathrm{mg}$ al día. En ese momento el paciente presentaba una descompensación importante de su diabetes tipo 2 e hipertensión, por lo que se motivó al paciente para que buscara tratamiento de la obesidad y comorbilidades metabólicas. El paciente fue evaluado por especialistas de nuestra Institución, quienes lo consideraron como candidato para una cirugía bariátrica, por lo que se incorporó a un programa multidisciplinario enfocado en cambiar hábitos de alimentación e iniciar actividad física. En enero de 2011 el paciente fue operado con éxito de un bypass gástrico en nuestro hospital, su Índice de Masa Corporal (IMC) preoperatorio era de 42,2 kg/ $\mathrm{m}^{2}$ y perímetro abdominal de $127 \mathrm{~cm}$.

A los 3 meses, el paciente fue atendido nuevamente en Dermatología por motivo de control de su psoriasis. Al examen físico no sólo constatamos una baja de peso considerable (IMC de $32,6 \mathrm{~kg} / \mathrm{m}^{2}$ ), si no que se encontraba completamente libre de lesiones cutáneas. Dada la resolución de sus lesiones, se decidió disminuir la dosis de Neotigasón ${ }^{\circledR}$ (a $25 \mathrm{mg}$ tres veces por semana), con el plan de suspenderlo de manera definitiva a futuro. Además, el paciente refirió una importante mejoría en su calidad de vida y autoestima.

\section{Discusión}

La asociación entre obesidad y psoriasis nació de estudios epidemiológicos europeos ${ }^{8,9}$. En 1986 un estudio escandinavo reveló mayor prevalencia de obesidad en mujeres psoriáticas que en el resto de las mujeres ${ }^{9}$. Más tarde Herron, confirmó dicha asociación en su estudio de cohorte al reportar una mayor incidencia de obesidad en los 500 pacientes con psoriasis que en el resto de la población del estado de Utah, siendo ambas poblaciones ajustadas por el IMC que presentaban antes del diagnóstico de la psoriasis ${ }^{10}$. Se identificó además que el riesgo de obesidad en los pacientes con psoriasis es directamente proporcional a la severidad de ésta (con un odds ratio [OR] de 1,8 para psoriasis severa en comparación con un OR de 1,3 para psoriasis moderada) ${ }^{11}$.

Por otra parte, se ha observado una mejoría de los síntomas de la psoriasis durante períodos de ayuno y menor ingesta calórica ${ }^{12}$. En un estudio clínico realizado en Croacia con 82 pacientes con psoriasis vulgar de 10 años de evolución y con tratamientos tópicos habituales, 40 de ellos recibieron alimentación hospitalaria común, mientras que 42 recibieron una dieta hipocalórica. Después de 4 semanas, el grupo con restricción calórica mostró una mejoría clínica estadísticamente significativa de las lesiones cutáneas en relación al grupo control ${ }^{13}$. En cuanto a pacientes con psoriasis severa en tratamiento con 
REMISIÓN DE LA PSORIASIS LUEGO DE BYPASS GÁSTRICO

Tabla 1. Resumen de los casos publicados en la literatura de pacientes con psoriasis operados de cirugía bariátrica

\begin{tabular}{|c|c|c|c|c|c|c|}
\hline Caso & $\begin{array}{c}\text { Edad } \\
\text { (Sexo) }\end{array}$ & $\begin{array}{c}\text { IMC } \\
\left(\mathrm{kg} / \mathrm{m}^{2}\right) \\
\text { T0 }\end{array}$ & $\begin{array}{l}\text { Tratamiento } \\
\text { T0 }\end{array}$ & $\begin{array}{l}\text { Tipo de } \\
\text { cirugía }\end{array}$ & $\begin{array}{c}\text { IMC } \\
\left(\mathrm{kg} / \mathrm{m}^{2}\right) \\
\text { T1 }\end{array}$ & Resultados y seguimiento \\
\hline $\begin{array}{l}\text { Hossler et } \mathrm{al}^{29} \\
\text { Caso } 1\end{array}$ & $\begin{array}{c}34 \\
(\mathrm{M})\end{array}$ & 52 & $\begin{array}{l}\text { Corticoide tópico, } \\
\text { fototerapia (UVB), } \\
\text { PUVA }\end{array}$ & $\begin{array}{l}\text { Bypass } \\
\text { gástrico }\end{array}$ & 40,8 & $\begin{array}{l}\text { Mejoría de la psoriasis. Tratamiento } \\
\text { intermitente con corticoide tópico. } \\
\text { Sin rebrote por } 6 \text { años de seguimiento }\end{array}$ \\
\hline $\begin{array}{l}\text { Hossler et } \mathrm{al}^{29} \\
\text { Caso } 2\end{array}$ & $\begin{array}{l}42 \\
(\mathrm{~F})\end{array}$ & 55,6 & $\begin{array}{l}\text { Terapia sistémica } \\
\text { con Etarnecept }\end{array}$ & $\begin{array}{l}\text { Bypass } \\
\text { gástrico }\end{array}$ & 37,3 & $\begin{array}{l}\text { Mejoría de la psoriasis. Sin rebrote en } \\
13 \text { meses de seguimiento }\end{array}$ \\
\hline $\begin{array}{l}\text { Higa-Sansone et } \mathrm{al}^{30} \\
\text { Caso } 3\end{array}$ & $\begin{array}{c}55 \\
(\mathrm{M})\end{array}$ & 41 & Corticoide tópico & $\begin{array}{l}\text { Bypass } \\
\text { gástrico }\end{array}$ & 29,1 & $\begin{array}{l}\text { Remisión de la psoriasis. Suspensión } \\
\text { definitiva del tratamiento. Sin rebrote } \\
\text { de lesiones en } 2 \text { años de seguimiento }\end{array}$ \\
\hline $\begin{array}{l}\text { De Menezes et } \mathrm{al}^{31} \\
\text { Caso } 1\end{array}$ & $\begin{array}{l}56 \\
(\mathrm{M})\end{array}$ & 46,9 & Corticoide tópico. & $\begin{array}{l}\text { Bypass } \\
\text { gástrico }\end{array}$ & 38,1 & $\begin{array}{l}\text { Remisión de la psoriasis. Suspen- } \\
\text { sión definitiva del tratamiento. Sin } \\
\text { rebrote de lesiones en } 4 \text { meses de } \\
\text { seguimiento }\end{array}$ \\
\hline $\begin{array}{l}\text { Pérez-Pérez et } \mathrm{al}^{32} \\
\text { Caso } 1\end{array}$ & $\begin{array}{l}52 \\
(\mathrm{~F})\end{array}$ & 56,9 & $\begin{array}{l}\text { Tratamiento tópico } \\
\text { y sistémico con } \\
\text { Efalizumab }\end{array}$ & $\begin{array}{l}\text { Bypass } \\
\text { gástrico }\end{array}$ & 31,9 & $\begin{array}{l}\text { Durante los } 3 \text { años de seguimiento } \\
\text { las lesiones se hicieron más severas. } \\
\text { Requirió fototerapia y tratamiento } \\
\text { sistémico con Infliximab }\end{array}$ \\
\hline
\end{tabular}

T0: pre-quirúrgico, T1: post-quirúrgico.

agentes biológicos, se ha reportado una mayor respuesta a los medicamentos en aquellos que logran adherir a una dieta hipocalórica y, por consiguiente, disminuir su $\mathrm{IMC}^{14}$. Se destaca que tan solo una disminución de apenas $5 \mathrm{~kg}$ de peso marca una diferencia estadística en la evolución positiva de la psoriasis ${ }^{15}$.

Para entender la relación entre la baja de peso y la evolución de la psoriasis, es necesario comprender los mecanismos fisiopatológicos que comparten ambas enfermedades. La obesidad corresponde a una enfermedad inflamatoria crónica de bajo grado, caracterizada por una elevación de los niveles plasmáticos de citoquinas proinflamatorias como el factor de necrosis tumoral alfa (TNF- $\alpha$ ), la interleuquina- 6 (IL-6) y proteínas de fase aguda, tales como la proteína $\mathrm{C}$ reactiva $(\mathrm{PCR})^{16}$. Esta condición es producida por los adipositos, ya que éstos se comunican con el resto del organismo mediante la secreción de las llamadas adipokinas, moléculas con actividad proinflamatoria, trombótica y vasoactiva ${ }^{17}$, dentro de las cuales destaca el TNF- $\alpha$, inhibidor del activador del plasminógeno 1, IL-6 y leptina. También se observa una disminución de la adiponectina, citoquina con actividad antiinflamatoria ${ }^{18}$. Por una parte, estas citoquinas actúan directamente en el hígado, estimulando la síntesis de proteínas inflamatorias ${ }^{19}$. Por otra, las citoquinas incrementan la lipólisis, es decir, el tejido adiposo libera constantemente ácidos grasos libres hacia la circulación periférica ${ }^{18}$, los cuales son capaces de aumentar el estrés oxidativo, y por lo tanto, el ambiente inflamatorio y la actividad vascular ${ }^{20}$. Cabe destacar que el tejido adiposo de predominio central se asocia a mayor cantidad de grasa visceral, comparado con la distribución periférica de ésta. Los adipositos de la grasa visceral son metabólicamente más activos, liberando mayor cantidad de citoquinas y ácidos grasos, por lo que se esperaría encontrar un ambiente más inflamatorio aún en los pacientes con obesidad abdominal ${ }^{21}$.

El estado inflamatorio asociado con la obesidad ha sido propuesto como nexo entre diversas condiciones patológicas que suelen coexistir, estado conocido como "Síndrome Metabólico"22. El efecto de las citoquinas sobre la sensibilidad insulínica a nivel hepático ${ }^{23}$ y muscular ${ }^{24}$ ha sido ampliamente estudiado. A diferencia de la psoriasis, en que su asociación con la obesidad se ha demostrado a nivel cínico sólo durante las últimas décadas. Siendo ambas patologías inflamatorias con un sustrato fisiopatológico común, parece razonable el hecho de que la baja de peso pueda contribuir de manera positiva a mejorar la evolución de estos pacientes.

La cirugía bariátrica ha demostrado ser el tratamiento más efectivo para bajar de peso en pacientes con obesidad mórbida (IMC mayor a $40 \mathrm{~kg} / \mathrm{m}^{2}$ ). La eficacia en la remisión de las comorbilidades y en la mantención del peso a largo plazo ${ }^{25-27}$, hace que este procedimiento sea actualmente el tratamiento de elección tanto para pacientes con obesidad mórbida 
como para aquellos que presentan un IMC sobre $35 \mathrm{~kg} / \mathrm{m}^{2}$ junto a comorbilidades secundarias a la obesidad $^{28}$.

En la literatura existe reporte de sólo 5 casos de pacientes con psoriasis que fueron operados de bypass gástrico ${ }^{29-32}$ (Tabla 1), cuatro de ellos presentaron una evolución favorable de la psoriasis luego de la pérdida de peso. Nosotros reportamos un nuevo caso de un paciente con diagnóstico de psoriasis moderada a severa -dado la necesidad de tratamiento sistémico- de larga data, que presentó una excelente evolución de su enfermedad luego de bajar de peso, lo cual se logró mediante tratamiento quirúrgico de su obesidad y comorbilidades metabólicas. A nuestro juicio, la psoriasis podría considerarse como una nueva comorbilidad cuya remisión es esperable luego de la cirugía bariátrica. Esperamos a futuro poder demostrar lo anterior con mayor número de pacientes, tambien con otras técnicas quirúrgicas (como la gastrectomía en manga), así como poder objetivar el impacto en la calidad de vida de estos pacientes.

\section{Agradecimientos}

A la Dra. Verónica Iribarra y al Dr. Ricardo Funke, pertenecientes al Departamento de Nutrición y al de Cirugía Digestiva de la Pontificia Universidad Católica de Chile, respectivamente. Ambos contribuyeron de manera importante al tratamiento de nuestro paciente.

\section{Referencias}

1. Naldi L. Epidemiology of psoriasis. Curr Drug Targets Inflamm Allergy. 2004;3:121-8.

2. Romero W, de la Cruz C. Recomendaciones para el uso de agentes biológicos en psoriasis. Rev Chi Dermatol. 2010;26:1-34.

3. Puig-Sanz L. [Psoriasis, a systemic disease?]. Actas Dermosifiliogr. 2007;98:396-402

4. Hamminga EA, van der Lely AJ, Newmann HAM, Thio HB. Chronic inflammation in psoriasis and obesity: Implications for therapy. Med Hypotheses 2006;67:786-73.

5. Bardazzi F, Balestri R, Balde E, Antonucci A, De Tommaso S, Patrizi A. Correlation between BMI and PASI in patients affected by moderate to severe psoriasis undergoing biological therapy. Dermatol Ther. 2010;23:S14-9.

6. Simons R. Additional studies on psoriasis in the tropics and in starvation camps. J Invest Dermatol. 1949;12:285-95

7. Farías MM, de la Cruz C, Serrano V. Psoriasis y obesidad: revisión y recomendaciones prácticas. Actas Dermosifiliogr. 2011; [Article in press].

8. Henseler T, Christophers E. Disease concomitance in psoriasis. J Am Acad Dermatol. 1995;32:982-6.
9. Lindegard B. Diseases associated with psoriasis in a general population of 159,200 middle-aged, urban, native Swedes. Dermatología 1986;172:298-304.

10. Heron M, Hinckley M, Hoffman M, Papenfuss J, Hansen C, Callis C, et al. Impact of obesity and smoking on psoriasis presentation and management. Arch Dermatol. 2005;141:1527-34.

11. Neimann A, Shin D, Wang X, Margolis D, Troxel A, Gelfand M. Prevalence of cardiovascular risk factors in patients with psoriasis. J Am Acad Dermatol. 2006;55:829-35.

12. Simons R. Additional studies on psoriasis in the tropics and in starvation camps. J Invest Dermatol. 1949;12:285-94

13. Rucevic I, Perl A, Barisic-Drusko V, Adam-Perl M. The role of the low energy diet in psoriasis vulgaris treatment. Coll Antropol. 2003;27:S41-8.

14. Gisondi P, Del Giglio M, Di Francesco V, Zamboni M, Girolomoni G. Weight loss improves the response of obese patients with moderate-to-severe chronic plaque psoriasis to low-dose cyclosporine therapy: a randomized, controlled, investigator-blinded clinical trial. Am J Clin Nutr. 2008;88:1242-7.

15. Bardazzi F, Balestri R, Balde E, Antonucci A, De Tommaso S, Patrizi A. Correlation between BMI and PASI in patients affected by moderate to severe psoriasis undergoing biological therapy. Dermatol Ther. 2010;23:S14-9.

16. Das U. Is obesity an inflammatory condition? Nutrition 2001;17:953-66.

17. Scherer P. Adipose tissue: from lipid storage compartment to endocrine organ. Diabetes 2006;55:1537-45.

18. Eckel R, Grundy S, Zimmet P. The metabolic syndrome. Lancet. 2005;365:1415-28.

19. Stolar M. Metabolic syndrome: controversial but useful. Cleve Clin J Med. 2007;74:199-202.

20. Tripathy D, Mohanty P, Dhindsa S, Syed T, Ghanim $\mathrm{H}$, Aljada A, et al. Elevation of free fatty acids induces inflammation and impairs vascular reactivity in healthy subjects. Diabetes 2003;52:2882-7.

21. Guo Z, Hensrud D, Johnson M, Jensen M. Regional postprandial fatty acid metabolism in different obesity phenotypes. Diabetes 1999;48:1586-92.

22. Rana J, Nieuwdorp M, Jukema J, Kastelein J. Cardiovascular metabolic syndrome - an interplay of, obesity, inflammation, diabetes and coronary heart disease. Diabetes Obes Metab. 2007;9:218-32.

23. Samuel V, Liu Z, Qu X, Elder B, Bilz S, Befroy D, et al. Mechanism of hepatic insulin resistance in non-alcoholic fatty liver disease. J Biol Chem. 2004;279:32345-53.

24. Wei Y, Chen K, Whaley-Connell A, Stump C, Ibdah J, Sowers J. Skeletal muscle insulin resistance: role of inflammatory cytokines and reactive oxygen species. Am J Physiol Regul Integr Comp Physiol. 2008;294:R67380.

25. Boza C, Gamboa C, Awruch D, Pérez G, Escalona A, 
Ibañez L. Laparoscopic Roux-en-Y gastric bypass versus laparoscopic adjustable gastric banding: five years of follow-up. Surg Obes Relat Dis. 2010;6:470-5

26. Sjöström L, Narbro K, Sjöström CD, Karason K, Larsson B, Wedel H, et al. Swedish Obese Subjects Study. Effects of bariatric surgery on mortality in Swedish obese subjects. N Engl J Med. 2007;357:741-52

27. Farias MM, Cuevas A, Rodriguez F. Set-point theory and obesity. Met Synd Relat Disord. 2011;9:85-9.

28. Colquitt J, Picot J, Loveman E, Clegg AJ. Surgery for obesity. Cochrane Database Syst Rev. 2009;2:CD003641.

29. Hossler E, Marron M, Moward C. Gastric bypass sur- gery improves psoriasis. Am Acad Dermatol. 2010; [E pub ahead of print]

30. Higa-Sansone G, Szomstein S, Soto F, Brasecsco O, Cohen C, Rosenthal R. Psoriasis remission after laparoscopic Roux-en-Y gastric bypass for morbid obesity. Obes Surg. 2004;14:1132-4.

31. de Menezes Ettinger JE, Azaro E, de Souza CA, dos Santos Filho PV, Mello CA, Neves M, et al. Remission of psoriasis after open gastric bypass. Obes Surg. 2006;16:94-7

32. Pérez-Pérez L, Allegue F, Caeiro JL, Zulaica JM. Severe psoriasis, morbid obesity and bariatric surgery. Clin Exp Dermatol. 2009;34:e421-2. 